\title{
AREA OF TRIANGLE IN THE PLANE WITH ALPHA DISTANCE FUNCTION
}

\author{
Chae Hee $\mathrm{OH}^{\mathrm{a}}$, Il SeOg Ko ${ }^{\mathrm{b}}$ And Byung HaK Kim ${ }^{\mathrm{c}, *}$
}

\begin{abstract}
The taxicab distance and Chinese-checker distance in the plane are practical distance notions with a wide range of applications compared to the Euclidean distance. The $\alpha$-distance was introduced as a generalization of these two distance functions. In this paper, we study alpha circle, trigonometry, and the area of a triangle in the plane with $\alpha$-distance.
\end{abstract}

\section{INTRODUCTION}

The Euclidean distance is the most frequently used when measuring the distance between two points on a plane. This distance notion is used so due to its simplicity to understand intuitively, but is not a useful way to move from one position to another in actuality. Consequently, the idea of how a taxi travels in modern cities was developed into a practical distance notion, the taxicab distance ([8]). The Chinese checker distance, on the other hand, mirrors the movement made by playing Chinese checkers, and was introduced by G. Chen et al. ([3]). The taxicab distance is closely related to movements in modern cities where most streets run at right angles with each other, and thus is more useful than the Euclidean distance. Since not all movements are made at right angles, however, the taxicab distance has been generalized into the $\alpha$-distance, and this includes the Chinese-checker distance as well ([12]).

Whereas there are many researches done in the taxicab geometry such as the distance between a point and line $([1],[3],[8])$, taxicab trigonometry ([2], [11], [13]), isoperimetric inequality ([9]) and some traits regarding triangle on taxicab plane

Received by the editors June 20, 2012. Accepted September 3, 2012.

2010 Mathematics Subject Classification. 51K05, 51M05.

Key words and phrases. alpha distance function, alpha trigonometry, triangle in alpha distance plane.

${ }^{*}$ Corresponding author. 
([7], [10], [13]), the alpha-distance is still on its basic steps with little research done including the generalization to $n$-dimensional case ([4], [5], [12]) and no further research yet. In this point of view, we define alpha circle and trigonometry, and study the area of a triangle in the alpha distance plane. These results and methods in this paper would lead to further studies in the relatively newly born field of geometry and help applications to real life.

\section{Alpha Distance and Alpha Circle}

Let $A\left(x_{1}, y_{1}\right)$ and $B\left(x_{2}, y_{2}\right)$ be two points in $\mathbb{R}^{2}$ and denote

$$
\Delta_{A B}=\max \left\{\left|x_{1}-x_{2}\right|,\left|y_{1}-y_{2}\right|\right\}, \quad \delta_{A B}=\min \left\{\left|x_{1}-x_{2}\right|,\left|y_{1}-y_{2}\right|\right\} .
$$

The Euclidean distance $d_{E}(A, B)$, the taxicab distance $d_{T}(A, B)$, and the Chinese checker distance $d_{C}(A, B)$ are defined by $([6],[8])$

$d_{E}(A, B)=\sqrt{\Delta_{A B}^{2}+\delta_{A B}^{2}}, d_{T}(A, B)=\Delta_{A B}+\delta_{A B}, d_{C}(A, B)=\Delta_{A B}+(\sqrt{2}-1) \delta_{A B}$, respectively. The generalization of $d_{T}$ and $d_{C}$, the alpha-distance $d_{\alpha}(A, B)$ was introduced by $([12])$

$$
d_{\alpha}(A, B)=\Delta_{A B}+(\sec \alpha-\tan \alpha) \delta_{A B} \text { for any } \alpha \in[0, \pi / 4] .
$$

These four distances all tie if $\delta_{A B}=0$, and if $\delta_{A B}>0$

$$
d_{E}(A, B)<d_{C}(A, B)<d_{\alpha}(A, B)<d_{T}(A, B) \text { for all } \alpha \in(0, \pi / 4)([1]) .
$$

Denote $c$ by $c=\tan \alpha-\sec \alpha$, and thus $-1 \leq c \leq 1-\sqrt{2}$.

Now consider alpha circle and its basic properties. We can define alpha circle as a set of points from a fixed alpha distance, the radius, from a fixed point, the center. That is, alpha circle with center $P$ and radius $r$ is $C_{\alpha}(P, r)=\left\{Q \in \mathbb{R}^{2} \mid d_{\alpha}(P, Q)=r\right\}$. Then we can see that the coordinate of the point where the alpha unit circle and the graph $y=x$ meets in the first quadrant is $\left(\frac{1}{1-c}, \frac{1}{1-c}\right)$. The angle that determines the shape of an alpha circle is given as below.

Proposition 2.1. The acute angle $\theta$ (measured in Euclidean angle) between an alpha circle segment and neighboring axis is $\theta=\frac{\pi}{4}+\frac{\alpha}{2}$.

Proof. Let $A$ be the point of intersection between the alpha unit circle and the $x$-axis, and $B$ the point in the first quadrant(see figure 1). Let $H$ be the foot of perpendicular of $B$ to the $x$-axis, and $P$ a point on the $x$-axis whose $x$-coordinate is smaller than that of $H$ and satisfies $\angle H B P=\alpha$. Then $1=d_{\alpha}(B, O)=d_{E}(B, P)+d_{E}(P, O)$, 
so $\triangle P A B$ is equilateral. Thus we obtain $\angle P A B=\angle P B A$, and $\theta=\angle B A O=$ $\frac{\pi}{4}+\frac{\alpha}{2}$.

In Figure $1, d_{\alpha}(A, B)=\frac{1+c^{2}}{1-c}$. The circumference of alpha unit circle is $\frac{8\left(1+c^{2}\right)}{1-c}$, and the diameter is 2 . Thus we have

Proposition 2.2. The circular constant of alpha circle is $\pi_{\alpha}=\frac{4\left(1+c^{2}\right)}{1-c}$.

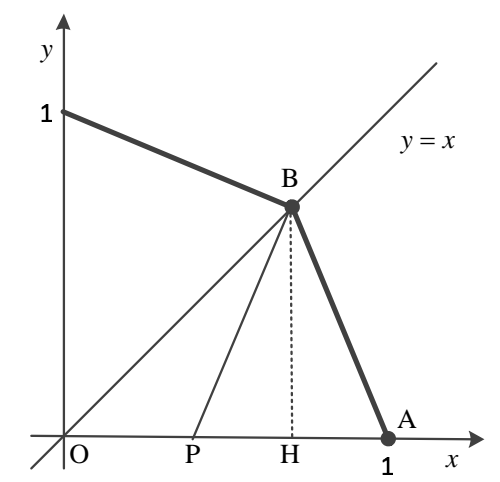

Figure 1: Alpha unit circle in the first quadrant.

Now we investigate the geometric meaning of alpha circle; why the line segment of an alpha unit circle satisfies to be a part of circle.

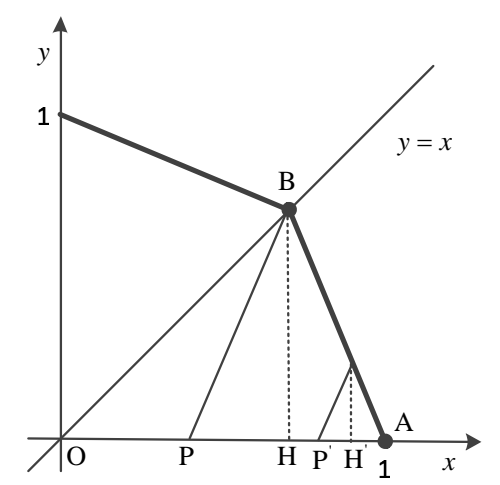

Figure 2: Geometric meaning of an alpha circle segment.

The alpha distance between the points $B$ and $O$ is 1 (see Figure 2). Set points $H$ and $P$ as we did in the proof of the Proposition 2.1. Then $1=d_{\alpha}(B, O)=$ $d_{E}(B, P)+d_{E}(P, O)$ and $\triangle P A B$ is equilateral.

Now let $V$ be any point on the alpha unit circle in the first quadrant and similarly set the points $H^{\prime}$ and $P^{\prime}$. Then $\overline{B P} / / \overline{V P^{\prime}}$, so $\Delta P^{\prime} A V$ is also equilateral which leads us to the conclusion that

$$
\left.d_{\alpha}(V, O)=d_{E}\left(V, P^{\prime}\right)+d_{E}\left(P^{\prime}, O\right)=1 \text { (for any point } V \text { on the } \overline{A B}\right) .
$$




\section{Alpha Angle and Trigonometry}

In this chapter, we define the alpha angle and alpha trigonometry with alpha circle introduced above, and find the value of trigonometric function for every alpha angle $\theta_{\alpha}$. We refer to the definition of the taxicab radian([11]) to make its alpha version work for any angle $\alpha$.

Definition 3.1. An alpha radian is an angle whose vertex is the center of an alpha unit circle and intercepts an arc of (alpha distance) length 1 . The measure of an alpha angle $\theta_{\alpha}$ is the number of alpha radians subtended by the angle on the alpha unit circle about the vertex.

Due to Definition 3.1. we see that the length $l$ of the arc intercepted on an alpha circle of radius $r$ by the central angle with alpha measure $\theta_{\alpha}$ is given by $l=r \theta_{\alpha}$. The reference angle of an angle $\theta_{\alpha}$ is the smallest angle between $\theta_{\alpha}$ and the $x$-axis. If the reference angle is equal to 0 , then the alpha angle is in standard position. Let $P(x, y)$ be the point of intersection of the terminal side of the alpha angle $\theta_{\alpha}$ in standard position with the alpha circle of radius $r$, then the standard alpha angle $\theta_{\alpha}$ is given by

$$
\theta_{\alpha}= \begin{cases}-c+\frac{c x+y}{r}, & \theta_{\alpha} \in I_{1} \\ \frac{c^{2}+c+2}{1-c}+\frac{-x-c y}{r}, & \theta_{\alpha} \in I_{2} \\ \frac{3 c^{2}-c+2}{1-c}+\frac{-x+c y}{r}, & \theta_{\alpha} \in I_{3} \\ \frac{3 c^{2}+c+4}{1-c}+\frac{c x-y}{r}, & \theta_{\alpha} \in I_{4} \\ \frac{5 c^{2}-c+4}{1-c}+\frac{-c x-y}{r}, & \theta_{\alpha} \in I_{5} \\ \frac{5 c^{2}+c+6}{1-c}+\frac{x+c y}{r}, & \theta_{\alpha} \in I_{6} \\ \frac{7 c^{2}-c+6}{1-c}+\frac{x-c y}{r}, & \theta_{\alpha} \in I_{7} \\ \frac{7 c^{2}+c+8}{1-c}+\frac{-c x+y}{r}, & \theta_{\alpha} \in I_{8}\end{cases}
$$

where $I_{n}=\left[\frac{(n-1) \pi_{\alpha}}{4}, \frac{n \pi_{\alpha}}{4}\right),(1 \leq n \leq 8)$.

Since the alpha distance function depends on the sizes of $|x|$ and $|y|$, the alpha angle also varies with every $\frac{\pi_{\alpha}}{4}$.

Definition 3.2. The point of intersection of the terminal side of the alpha angle $\theta_{\alpha}$ in standard position with the alpha unit circle is the point $\left(\cos \theta_{\alpha}, \sin \theta_{\alpha}\right)$, and $\tan \theta_{\alpha}=\frac{\sin \theta_{\alpha}}{\cos \theta_{\alpha}}$.

By use of Definition 3.2, we can easily show that 


$$
\left\{\begin{array}{l}
\left|\sin \theta_{\alpha}\right|-c\left|\cos \theta_{\alpha}\right|=1,\left|\sin \theta_{\alpha}\right| \geq\left|\cos \theta_{\alpha}\right| \\
\left|\cos \theta_{\alpha}\right|-c\left|\sin \theta_{\alpha}\right|=1,\left|\sin \theta_{\alpha}\right| \leq\left|\cos \theta_{\alpha}\right|
\end{array} .\right.
$$

It is easy to verify that alpha sine function and cosine function are both period function with period $2 \pi_{\alpha}$, and tangent function has period $\pi_{\alpha}$. Then we can define the trigonometry in alpha distance geometry as below.

$$
\cos \theta_{\alpha}=\left\{\begin{array}{ll}
1+\frac{c}{c^{2}+1} \theta_{\alpha}, & \theta_{\alpha} \in I_{1} \\
\frac{2}{1-c}-\frac{1}{c^{2}+1} \theta_{\alpha}, & \theta_{\alpha} \in I_{2}, I_{3} \\
\frac{c}{c^{2}+1} \theta_{\alpha}-\frac{3 c+1}{1-c}, & \theta_{\alpha} \in I_{4} \\
\frac{5 c-1}{1-c}-\frac{c}{c^{2}+1} \theta_{\alpha}, & \theta_{\alpha} \in I_{5} \\
\frac{1}{c^{2}+1} \theta_{\alpha}-\frac{6}{1-c}, & \theta_{\alpha} \in I_{6}, I_{7} \\
\frac{7 c+1}{1-c}-\frac{c}{c^{2}+1} \theta_{\alpha}, & \theta_{\alpha} \in I_{8}
\end{array} .\right.
$$

$$
\sin \theta_{\alpha}= \begin{cases}\frac{1}{c^{2}+1} \theta_{\alpha}, & \theta_{\alpha} \in I_{1} \\ \frac{c+1}{1-c}-\frac{c}{c^{2}+1} \theta_{\alpha}, & \theta_{\alpha} \in I_{2} \\ \frac{1-3 c}{1-c}+\frac{c}{c^{2}+1} \theta_{\alpha}, & \theta_{\alpha} \in I_{3} \\ \frac{4}{1-c}-\frac{1}{c^{2}+1} \theta_{\alpha}, & \theta_{\alpha} \in I_{4}, I_{5} \\ \frac{c}{c^{2}+1} \theta_{\alpha}-\frac{5 c+1}{1-c}, & \theta_{\alpha} \in I_{6} \\ \frac{7 c-1}{1-c}-\frac{c}{c^{2}+1} \theta_{\alpha}, & \theta_{\alpha} \in I_{7} \\ \frac{1}{c^{2}+1} \theta_{\alpha}-\frac{8}{1-c}, & \theta_{\alpha} \in I_{8}\end{cases}
$$

$$
\tan \theta_{\alpha}= \begin{cases}\frac{\theta_{\alpha}}{c \theta_{\alpha}+c^{2}+1}, & \theta_{\alpha} \in I_{1} \\ \frac{\left(c^{2}-c\right) \theta_{\alpha}+\left(c^{3}+c^{2}+c+1\right)}{(c-1) \theta_{\alpha}+2\left(c^{2}+1\right)}, & \theta_{\alpha} \in I_{2} \\ \frac{\left(c-c^{2}\right) \theta_{\alpha}+\left(-3 c^{3}+c^{2}-3 c+1\right)}{(c-1) \theta_{\alpha}+2\left(c^{2}+1\right)}, & \theta_{\alpha} \in I_{3} \\ \frac{(c-1) \theta_{\alpha}+4\left(c^{2}+1\right)}{\left(c-c^{2}\right) \theta_{\alpha}-\left(3 c^{3}+c^{2}+3 c+1\right)}, & \theta_{\alpha} \in I_{4} \\ \frac{(c-1) \theta_{\alpha}+4\left(c^{2}+1\right)}{\left(c^{2}-c\right) \theta_{\alpha}+\left(5 c^{3}-c^{2}+5 c-1\right)}, & \theta_{\alpha} \in I_{5} \\ \frac{\left(c-c^{2}\right) \theta_{\alpha}-\left(5 c^{3}+c^{2}+5 c+1\right)}{(1-c) \theta_{\alpha}-6\left(c^{2}+1\right)}, & \theta_{\alpha} \in I_{6} \\ \frac{\left(c^{2}-c\right) \theta_{\alpha}+\left(7 c^{3}-c^{2}+7 c-1\right)}{(1-c) \theta_{\alpha}-6\left(c^{2}+1\right)}, & \theta_{\alpha} \in I_{7} \\ \frac{(1-c) \theta_{\alpha}-8\left(c^{2}+1\right)}{\left(c^{2}-c\right) \theta_{\alpha}+\left(7 c^{3}+c^{2}+7 c+1\right)}, & \theta_{\alpha} \in I_{8}\end{cases}
$$




\section{Area of a Triangle in the Plane with Alpha Distance FUNCTION}

In this chapter, we consider the area of a triangle using alpha trigonometry when the alpha distances of two sides and the included alpha angle are given. To compute the area of a triangle, we place the vertex with the given included angle at the origin $O$. Note that the area of the triangle is invariant under the parallel translation. Let $\theta_{1}$ and $\theta_{2}$ denote the alpha angles between $x$-axis and the two sides of given length with $\theta_{1}<\theta_{2}$, and $\overline{A O}$ be one side with angle $\theta_{1}$, and $\overline{B O}$ be the other side. The length of sides are denoted $a$ and $b$, also in alpha version. Then we obtain

Theorem 4.1. The area of a triangle in the alpha plane is

$$
S=\frac{1}{2} a b\left|\sin \theta_{1} \cos \theta_{2}-\cos \theta_{1} \sin \theta_{2}\right| \text { (with alpha trigonometry). }
$$

Proof. Using the alpha trigonometry, we see that the coordinates of vertices $A$ and $B$ are given by $\left(a \cos \theta_{1}, a \sin \theta_{1}\right)$ and $\left(b \cos \theta_{2}, b \sin \theta_{2}\right)$ respectively. The extension of $\overline{A B}$ intersects any axis, say $x$-axis, and let $X$ be the point of intersection. Then the $x$-coordinate of $X$ is

$$
\frac{a b\left(\sin \theta_{1} \cos \theta_{2}-\cos \theta_{1} \sin \theta_{2}\right)}{a \sin \theta_{1}-b \sin \theta_{2}},
$$

which leads us to the conclusion that $S=\frac{1}{2} a b\left|\sin \theta_{1} \cos \theta_{2}-\cos \theta_{1} \sin \theta_{2}\right|$.

Now we study how the area of a triangle acts as the angles $\theta_{1}$ and $\theta_{2}$ vary.

Let $I_{n}=\left[\frac{(n-1) \pi_{\alpha}}{4}, \frac{n \pi_{\alpha}}{4}\right)$ for $n=1,2, \cdots, 8$, and $(i, j)$ denotes $\theta_{1} \in I_{i}$ and $\theta_{2} \in I_{j}$.

Depending on which interval the angles $\theta_{1}$ and $\theta_{2}$ are in, there are 64 possible cases of triangles in the alpha plane. But we can reduce those cases into eleven of them, as $\frac{\pi}{2}$-rotated one is considered to be same. An equal sign between ordered pairs denotes those pairs are considered same, and then we have eleven cases as below

$$
\begin{aligned}
& (1,1)=(4,4)=(5,5)=(8,8) \\
& (2,2)=(3,3)=(6,6)=(7,7) \\
& (1,2)=(3,4)=(5,6)=(7,8) \\
& (1,8)=(4,5) \\
& (2,3)=(6,7) \\
& (1,3)=(1,7)=(2,4)=(2,8)=(3,5)=(4,6)=(5,7)=(6,8)
\end{aligned}
$$




$$
\begin{aligned}
& (1,4)=(5,8) \\
& (3,6)=(2,7) \\
& (1,6)=(2,5)=(3,8)=(4,7) \\
& (1,5)=(4,8) \\
& (2,6)=(3,7)
\end{aligned}
$$

and consider the ones on the left end. For each case, we substitute trigonometry formula (1),(2) and (3) for corresponding terms in equation (4) to obtain specific value of the area of a triangle.

Case of $(1,1): \theta_{1}, \theta_{2} \in I_{1}$

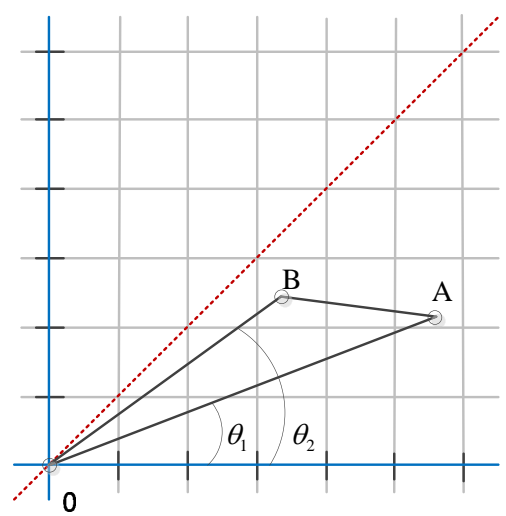

Figure 3: $\theta_{1}, \theta_{2} \in I_{1}$

$$
\begin{aligned}
S & =\frac{1}{2} a b\left(\cos \theta_{1} \sin \theta_{2}-\sin \theta_{1} \cos \theta_{2}\right) \\
& =\frac{1}{2} a b\left\{\left(1+\frac{c}{c^{2}+1} \theta_{1}\right) \frac{1}{c^{2}+1} \theta_{2}-\frac{1}{c^{2}+1} \theta_{1}\left(1+\frac{c}{c^{2}+1} \theta_{2}\right)\right\} \\
& =\frac{1}{2} a b\left(\frac{\theta_{2}-\theta_{1}}{c^{2}+1}\right) \\
& =\frac{1}{2} a b \sin \left(\theta_{2}-\theta_{1}\right)
\end{aligned}
$$

Case of $(1,2): \theta_{1} \in I_{1}, \theta_{2} \in I_{2}$

$$
\begin{aligned}
S & =\frac{1}{2} a b\left(\cos \theta_{1} \sin \theta_{2}-\sin \theta_{1} \cos \theta_{2}\right) \\
& =\frac{1}{2} a b\left\{\left(1+\frac{c}{c^{2}+1} \theta_{1}\right)\left(\frac{1+c}{1-c}-\frac{c}{c^{2}+1} \theta_{2}\right)-\frac{1}{c^{2}+1} \theta_{1}\left(\frac{2}{1-c}-\frac{1}{c^{2}+1} \theta_{2}\right)\right\}
\end{aligned}
$$

Case of $(1,5): \theta_{1} \in I_{1}, \theta_{2} \in I_{5}$

$$
\begin{aligned}
S & =\frac{1}{2} a b\left(\cos \theta_{1} \sin \theta_{2}-\sin \theta_{1} \cos \theta_{2}\right) \\
& =\frac{1}{2} a b\left\{\left(1+\frac{c}{c^{2}+1} \theta_{1}\right)\left(\frac{4}{1-c}-\frac{1}{c^{2}+1} \theta_{2}\right)-\frac{1}{c^{2}+1} \theta_{1}\left(\frac{5 c-1}{1-c}-\frac{c}{c^{2}+1} \theta_{2}\right)\right\} \\
& =\frac{1}{2} a b\left\{\frac{4}{1-c}-\frac{1}{c^{2}+1}\left(\theta_{2}-\theta_{1}\right)\right\} \\
& =\frac{1}{2} a b \sin \left(\theta_{2}-\theta_{1}\right)
\end{aligned}
$$


Case of $(2,6): \theta_{1} \in I_{2}, \theta_{2} \in I_{6}$

$$
\begin{aligned}
S= & \frac{1}{2} a b\left(\cos \theta_{1} \sin \theta_{2}-\sin \theta_{1} \cos \theta_{2}\right) \\
= & \frac{1}{2} a b\left\{\left(\frac{2}{1-c}-\frac{1}{c^{2}+1} \theta_{1}\right)\left(\frac{c}{c^{2}+1} \theta_{2}-\frac{5 c+1}{1-c}\right)\right. \\
& \left.\quad-\left(\frac{c+1}{1-c}-\frac{c}{c^{2}+1} \theta_{1}\right)\left(\frac{1}{c^{2}+1} \theta_{2}-\frac{6}{1-c}\right)\right\} \\
= & \frac{1}{2} a b\left\{\frac{4}{1-c}+\frac{1}{c^{2}+1}\left(\theta_{1}-\theta_{2}\right)\right\} \\
= & \frac{1}{2} a b \sin \left(\theta_{2}-\theta_{1}\right)
\end{aligned}
$$

Case of $(1,3): \theta_{1} \in I_{1}, \theta_{2} \in I_{3}$

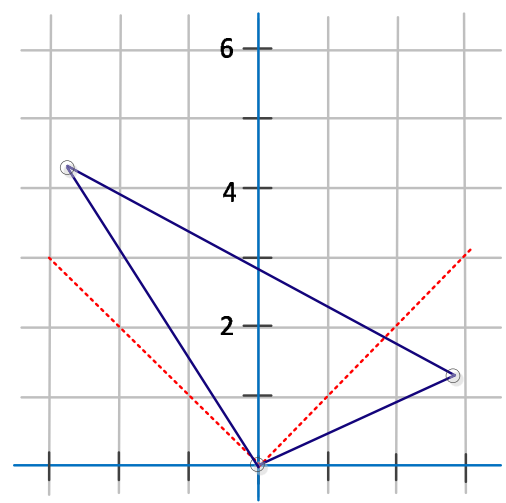

Figure 4: $\theta_{1} \in I_{1}, \theta_{2} \in I_{3}$

$\theta_{2}-\theta_{1} \in I_{3}$

$$
\begin{aligned}
S & =\frac{1}{2} a b\left(\cos \theta_{1} \sin \theta_{2}-\sin \theta_{1} \cos \theta_{2}\right) \\
& =\frac{1}{2} a b\left\{\left(1+\frac{c}{c^{2}+1} \theta_{1}\right)\left(\frac{1-3 c}{1-c}+\frac{c}{c^{2}+1} \theta_{2}\right)-\frac{1}{c^{2}+1} \theta_{1}\left(\frac{2}{1-c}-\frac{1}{c^{2}+1} \theta_{2}\right)\right\} \\
& =\frac{1}{2} a b\left\{\sin \left(\theta_{2}-\theta_{1}\right)-\left(c^{2}+1\right) \cos \theta_{2} \sin \theta_{1}\right\}
\end{aligned}
$$

$\theta_{2}-\theta_{1} \in I_{2}$

$$
\begin{aligned}
S & =\frac{1}{2} a b\left(\cos \theta_{1} \sin \theta_{2}-\sin \theta_{1} \cos \theta_{2}\right) \\
& =\frac{1}{2} a b\left\{\left(1+\frac{c}{c^{2}+1} \theta_{1}\right)\left(\frac{1-3 c}{1-c}+\frac{c}{c^{2}+1} \theta_{2}\right)-\frac{1}{c^{2}+1} \theta_{1}\left(\frac{2}{1-c}-\frac{1}{c^{2}+1} \theta_{2}\right)\right\}
\end{aligned}
$$

Case of $(2,3): \theta_{1} \in I_{2}, \theta_{2} \in I_{3}$

$$
\begin{aligned}
S= & \frac{1}{2} a b\left(\cos \theta_{1} \sin \theta_{2}-\sin \theta_{1} \cos \theta_{2}\right) \\
= & \frac{1}{2} a b\left\{\left(\frac{2}{1-c}-\frac{1}{c^{2}+1} \theta_{1}\right)\left(\frac{1-3 c}{1-c}+\frac{c}{c^{2}+1} \theta_{1}\right)\right. \\
& \left.\quad-\left(\frac{1+c}{1-c}-\frac{c}{c^{2}+1} \theta_{1}\right)\left(\frac{2}{1-c}-\frac{1}{c^{2}+1} \theta_{2}\right)\right\} \\
= & \frac{1}{2} a b\left\{\frac{-8 c}{(1-c)^{2}}+\frac{5 c-1}{\left(c^{2}+1\right)(1-c)} \theta_{1}+\frac{3 c+1}{\left(c^{2}+1\right)(1-c)} \theta_{2}-\frac{2 c}{\left(c^{2}+1\right)^{2}} \theta_{1} \theta_{2}\right\}
\end{aligned}
$$


Case of $(1,4): \theta_{1} \in I_{2}, \theta_{2} \in I_{4}$

$$
\begin{aligned}
\theta_{2}-\theta_{2} & \in I_{4} \\
S & =\frac{1}{2} a b\left(\cos \theta_{1} \sin \theta_{2}-\sin \theta_{1} \cos \theta_{2}\right) \\
& =\frac{1}{2} a b\left\{\left(1+\frac{c}{c^{2}+1} \theta_{1}\right)\left(\frac{4}{1-c}-\frac{1}{c^{2}+1} \theta_{2}\right)-\frac{1}{c^{2}+1} \theta_{1}\left(\frac{c}{c^{2}+1} \theta_{2}-\frac{3 c+1}{1-c}\right)\right\} \\
& =\frac{1}{2} a b\left\{\sin \left(\theta_{2}-\theta_{1}\right)+\frac{c}{c^{2}+1}\left(\frac{7}{1-c} \theta_{1}-\frac{2}{c^{2}+1} \theta_{2}\right)\right\} \\
\theta_{2}-\theta_{1} & \in I_{3} \\
S & =\frac{1}{2} a b\left(\cos \theta_{1} \sin \theta_{2}-\sin \theta_{1} \cos \theta_{2}\right) \\
& =\frac{1}{2} a b\left\{\left(1+\frac{c}{c^{2}+1} \theta_{1}\right)\left(\frac{4}{1-c}-\frac{1}{c^{2}+1} \theta_{2}\right)-\frac{1}{c^{2}+1} \theta_{1}\left(\frac{c}{c^{2}+1} \theta_{2}-\frac{3 c+1}{1-c}\right)\right\}
\end{aligned}
$$

Case of $(2,2): \theta_{1}, \theta_{2} \in I_{2}$

$$
\begin{aligned}
S= & \frac{1}{2} a b\left(\cos \theta_{1} \sin \theta_{2}-\sin \theta_{1} \cos \theta_{2}\right) \\
= & \frac{1}{2} a b\left\{\left(\frac{2}{1-c}-\frac{1}{c^{2}+1} \theta_{1}\right)\left(\frac{1+c}{1-c}-\frac{c}{c^{2}+1} \theta_{2}\right)\right. \\
& \left.\quad \quad-\left(\frac{1+c}{1-c}-\frac{c}{c^{2}+1} \theta_{1}\right)\left(\frac{2}{1-c}-\frac{1}{c^{2}+1} \theta_{2}\right)\right\} \\
= & \frac{1}{2} a b \sin \left(\theta_{2}-\theta_{1}\right)
\end{aligned}
$$

Case of $(1,8): \theta_{1} \in I_{1}, \theta_{2} \in I_{8}$

$$
\begin{aligned}
\theta_{1}+\theta_{2} & \in I_{8} \\
S & =\frac{1}{2} a b\left(\sin \theta_{1} \cos \theta_{2}-\cos \theta_{1} \sin \theta_{2}\right) \\
& =\frac{1}{2} a b\left\{\frac{1}{c^{2}+1} \theta_{1}\left(\frac{7 c+1}{1-c}-\frac{c}{c^{2}+1} \theta_{2}\right)-\left(1+\frac{c}{c^{2}+1} \theta_{1}\right)\left(\frac{1}{c^{2}+1} \theta_{2}-\frac{8}{1-c}\right)\right\} \\
& =\frac{1}{2} a b \sin \left(\theta_{1}+\theta_{2}\right) \\
\theta_{1}+\theta_{2} & \in I_{1} \\
S & =\frac{1}{2} a b\left(\sin \theta_{1} \cos \theta_{2}-\cos \theta_{1} \sin \theta_{2}\right) \\
& =\frac{1}{2} a b\left\{\frac{1}{c^{2}+1} \theta_{1}\left(\frac{7 c+1}{1-c}-\frac{c}{c^{2}+1} \theta_{2}\right)-\left(1+\frac{c}{c^{2}+1} \theta_{1}\right)\left(\frac{1}{c^{2}+1} \theta_{2}-\frac{8}{1-c}\right)\right\} \\
& =\frac{1}{2} a b\left\{\sin \left(\theta_{1}+\theta_{2}\right)-\frac{8}{1-c}\right\}
\end{aligned}
$$

Case of $(1,6): \theta_{1} \in I_{1}, \theta_{2} \in I_{6}$

$$
\begin{aligned}
\theta_{2}-\theta_{1} & \in I_{6} \\
S & =\frac{1}{2} a b\left(\cos \theta_{1} \sin \theta_{2}-\sin \theta_{1} \cos \theta_{2}\right) \\
& =\frac{1}{2} a b\left\{\left(1+\frac{c}{c^{2}+1} \theta_{1}\right)\left(\frac{c}{c^{2}+1} \theta_{2}-\frac{5 c+1}{1-c}\right)-\frac{1}{c^{2}+1} \theta_{1}\left(\frac{1}{c^{2}+1} \theta_{2}-\frac{6}{1-c}\right)\right\} \\
& =\frac{1}{2} a b\left\{\sin \left(\theta_{2}-\theta_{1}\right)+\left(c^{2}-1\right) \sin \theta_{1} \cos \theta_{2}\right\} \\
\theta_{2}-\theta_{1} & \in I_{5} \\
S & =\frac{1}{2} a b\left(\cos \theta_{1} \sin \theta_{2}-\sin \theta_{1} \cos \theta_{2}\right) \\
& =\frac{1}{2} a b\left\{\left(1+\frac{c}{c^{2}+1} \theta_{1}\right)\left(\frac{c}{c^{2}+1} \theta_{2}-\frac{5 c+1}{1-c}\right)-\frac{1}{c^{2}+1} \theta_{1}\left(\frac{1}{c^{2}+1} \theta_{2}-\frac{6}{1-c}\right)\right\}
\end{aligned}
$$


Case of $(3,6): \theta_{1} \in I_{3}, \theta_{2} \in I_{6}$

$$
\begin{aligned}
S= & \frac{1}{2} a b\left(\sin \theta_{1} \cos \theta_{2}-\cos \theta_{1} \sin \theta_{2}\right) \\
= & \frac{1}{2} a b\left\{\left(\frac{1-3 c}{1-c}+\frac{c}{c^{2}+1} \theta_{1}\right)\left(\frac{1}{c^{2}+1} \theta_{2}-\frac{6}{1-c}\right)\right. \\
& \left.\quad-\left(\frac{2}{1-c}-\frac{1}{c^{2}+1} \theta_{1}\right)\left(\frac{c}{c^{2}+1} \theta_{2}-\frac{5 c+1}{1-c}\right)\right\} \\
= & \frac{1}{2} a b\left\{\frac{28 c-4}{(1-c)^{2}}-\frac{11 c+1}{\left(c^{2}+1\right)(1-c)} \theta_{1}-\frac{5 c-1}{\left(c^{2}+1\right)(1-c)} \theta_{2}+\frac{2 c}{\left(c^{2}+1\right)^{2}} \theta_{1} \theta_{2}\right\}
\end{aligned}
$$

\section{REFERENCES}

1. Z. Akca \& R. Kaya: On the Distance Formulae in three Dimensional Taxicab Space. Hadronic J. 27 (2004), 521-532.

2. A. Bayar \& R. Kaya: On a Taxicab Distance on a Sphere. Missouri J. Math. Sci. 17 (2005), 41-51.

3. G. Chen: Lines and Circles in Taxicab Geometry. Master Thesis, Department of Mathematics and Computer Sciences, Central Missouri State University (1992).

4. O. Gelisgen \& R. Kaya: Generalization of $\alpha$-distance to $n$-dimensional space. Scientific and Professional Journal of the Croatian Society for Geometry and Graphics (KoG). 10 (2006), 33-35.

5. __ : On $\alpha_{i}$-distance in $n$-dimensional space. Appl. Sci.(APPS). 10 (2008), 88-94.

6. O. Gelisgen, R. Kaya \& M. Ozcan: Distance formulae in the Chinese checker space. Int. J. Pure Appl. Math.. 26 (2006), no. 1, 35-44.

7. R. Kaya \& H.B. Colakoglu: Taxicab Versions of some Euclidean Theorems. Int. J. Pure Appl. Math.. 26 (2006), no. 1, 69-81.

8. E.F. Krause: Taxicab Geometry. Addison-Wesley Publishing Company, Menlo Park, 1975.

9. K.M. Kwak, S.M. Baik, W.S. Choi, J.B. Choi, I.S. Ko \& B.H. Kim: On the plane geometry using the taxicab distance function. J. Korean Soc. Math. Ed. Ser. E: Commun. Math. Educ.. 24 (2010), no. 3, 659-989.

10. M. Ozcan \& R. Kaya: Area of a triangle in terms of the taxicab distance. Missouri J. Math. Sci.. 15 (2003), 178-185

11. K. Thompson \& T. Dray: Taxicab angles and trigonometry. The Pi Mu Epsilon Journal. 11 (2000), no. 2, 87-96.

12. S. Tian: Alpha-distance-a generalization of Chinese checker distance and taxicab distance. Missouri J. Math. Sci.. 17 (2005), no. 1, 35-40.

13. S. Tian, S.S. So \& G. Chen: Concerning circles in taxicab geometry. Int. J. Math. Educ. Sci. Tech.. 28 (1997), no. 5, 727-733. 
${ }^{\mathrm{b}}$ GyeongGi Science High School, Suwon 440-800, Korea

Email address: iskois@naver.com

${ }^{c}$ Department of Applied Mathematics, College of Applied Science, Kyung Hee UniverSITY, SUWON 446-701, KorEA

Email address: bhkim@khu.ac.kr 